\title{
Strong Robustness in Multi-Phase Adaptive Control: the basic Scheme
}

\author{
Maria Cadic and Jan Willem Polderman \\ Faculty of Mathematical Sciences \\ University of Twente, P.O. Box 217 \\ 7500 AE Enschede, The Netherlands
}

\begin{abstract}
This paper introduces the general structure of adaptive control systems based on strong robustness. This adaptive approach splits into two phases. In the first phase, effort is put on identification until enough information is obtained in order to design a controller stabilizing the actual system. This is achieved if the input sequence is computed in such a way that the uncertainty on the system parameters to be controlled decreases sufficiently fast. Then, in the second phase, emphasis is shifted to control.
\end{abstract}

\section{Introduction}

The majority of adaptive control algorithms is based on the certainty equivalence principle [1], [8]. In each iteration of these algorithms, a new model of the unknown true system is computed from the previous model and measured data, and a new controller is designed on the basis of this model. However, due to the model uncertainty, there is little reason to believe that the controller based on this model leads to a stable closed-loop system. Therefore, the drawback of this approach is that we can expect some undesired transients to arise in the closed-loop system behavior.

To avoid bad transients, we propose to design a test checking on-line if the model-based controller stabilizes the true system. We would apply the controller to the system only in the case the closed-loop stability is guaranteed. However to design a test depending on the true system is not realistic since this system is not completely known. Instead, we propose to add a test such that at each time the uncertainty set is updated, it is checked whether any model taken from this set yields a controller that stabilizes any other system in the set. In particular, since the uncertainty set contains the true system to be controlled, this would guarantee that any model taken from this set stabilizes the true system. If the uncertainty set has this property, it is said to be strongly robust.

To this end we propose to construct an adaptive control system based on two phases. In the first phase, emphasis is put on identification until the identified uncertainty set is strongly robust. When strong robustness is achieved, it guarantees that any model from the uncertainty set leads to a controller stabilizing the true system. The adaptive system is then allowed to switch to the second phase where the effort is shifted to control, using a certainty equivalence routine just as in classical adaptive control. 
In this paper, emphasis is put on the description of the general structure of the adaptive control scheme based on strong robustness, irrespective of computational issues. These are addressed in [2] and [3]. We proceed as follows. In the next section we define the problem set-up. In Section 3 we introduce the general structure of the adaptive control system based on strong robustness. Further, we investigate some aspects of the analysis of the algorithm in Section 4. Next, we address some computational issues in Section 5. Then, in Section 6, we see to what extent the algorithm depends on the choice of the desired control objective, what brings us to introduce the notion of weak strong robustness. In Section 7, we modify the notion of strongly robust sets defined in Section 3 so that the time variations of the control system are also taken into account. Finally, we conclude in Section 8.

\section{General Set-Up of the problem}

We first introduce the class of systems to be controlled.

Assumption 2.1 (Systems). $\mathcal{P}_{n}$ denotes the class of linear time-invariant SISO systems which are open-loop stable and controllable described by

$$
y(k)=\phi^{T}(k) \theta^{0}+\delta(k), k \geq 0
$$

with a known order $n \geq 1$. $\phi(k)$ represents the regressor vector given by

$$
\begin{gathered}
\phi(k)=(-y(k-1), \cdots,-y(k-n), u(k-1), \cdots, u(k-n))^{T} \in \mathbb{R}^{2 n} . \\
\text { Here, } \theta^{0}=\left(a_{n-1}^{0}, \cdots, a_{0}^{0}, b_{n-1}^{0}, \cdots, b_{0}^{0}\right)^{T} \in \mathbb{R}^{2 n}
\end{gathered}
$$

denotes the unknown parameter vector. $\delta(k)$ represents the unknown and bounded measurement error, with a known bound d, i.e., $|\delta(k)| \leq d, k \geq 0$.

Remark 2.1. Assumption 2.1 requires that the system to be controlled is open-loop stable. This condition is motivated by our wish to apply open-loop identification in the first phase. Note that rather than to stabilize the real plant, our objective is to improve the control performance of the classical adaptive control system, in particular its transient behavior. Note also that the class of unstable plants for which a stabilizing controller is known can be included in our framework, considering then the closed-loop system to be the initial stable plant.

The control objective is left unspecified. Instead, we make the following assumption.

Assumption 2.2 (Controllers). For any system $\theta \in \mathcal{P}_{n}$, there exists a unique controller denoted by $\phi(\theta)$ leading to an asymptotically stable system and such that the control objective is satisfied. We moreover suppose that the map $\phi$ assigning to any system $\theta \in \mathcal{P}_{n}$ its controller $\phi(\theta)$ is a continuous map.

Remark 2.2. Continuity is essential for the existence of strongly robust sets of systems [2].

Remark 2.3. Pole Placement and LQ Control satisfy Assumption 2.2. 


\section{Multi-Phase Adaptive Control Systems based on Strong Robustness: the general structure}

This section presents the structure of the multi-phase adaptive control systems based on strong robustness.

\subsection{Block diagram}

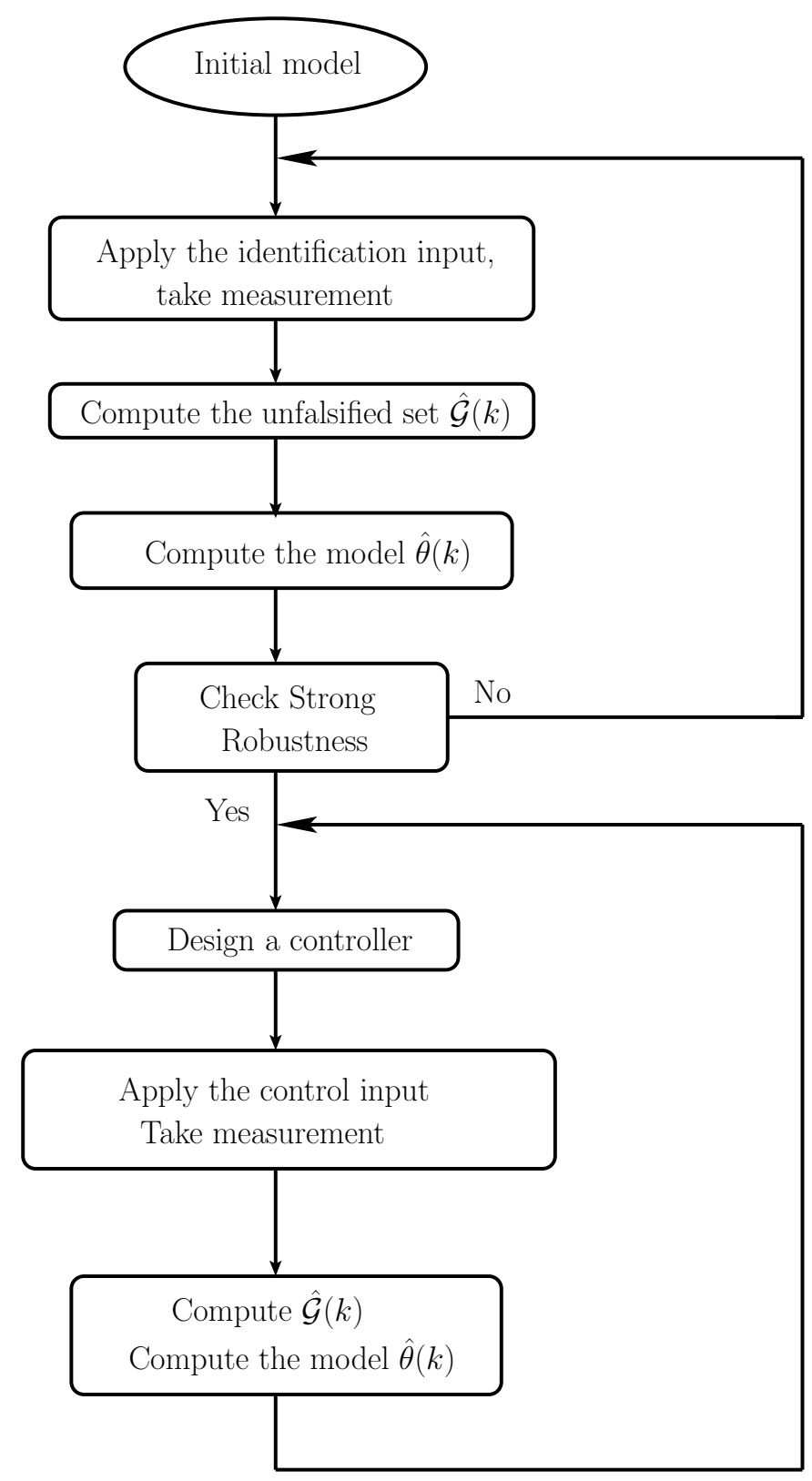

Figure 1: Iterative scheme. 
Figure 1 displays the block diagram of the proposed adaptive control scheme in which two phases are distinguished. In the first phase, at each new i/o data measurement, we first compute the set of all candidates for the parameters describing the system, we then select in this set a parameter vector to be the updated model and we finally test whether or not this set is strongly robust. If this test is positive, we switch to the second phase where the effort is put on control.

\subsection{Description}

We now give a brief description of the main components in Figure 1.

\subsubsection{Learning phase}

Compute the unfalsified set $\hat{\mathcal{G}}(k)$ : the unfalsified set $\hat{\mathcal{G}}(k)$ is defined as the set of parameter vectors $\theta$ consistent with the i/o data measurements up to time $k$ and the amplitude measurement error constraint $d$. Formally, $\hat{\mathcal{G}}(k)$ is the polyhedron defined by

$$
\hat{\mathcal{G}}(k)=\left\{\theta:\left|y(i)-\phi^{T}(i) \theta\right| \leq d, \forall i \leq k\right\}
$$

Denoting by $\mathcal{G}(k)$ the set of parameter vectors consistent with the i/o data at time $k$ :

$$
\mathcal{G}(k)=\left\{\theta:\left|y(k)-\phi^{T}(k) \theta\right| \leq d\right\}
$$

$\hat{\mathcal{G}}(k)$ can be thus defined by:

$$
\hat{\mathcal{G}}(k)=\mathcal{G}(k) \cap \hat{\mathcal{G}}(k-1)=\bigcap_{i \leq k} \mathcal{G}(i) .
$$

Compute the model $\hat{\theta}(k)$ : the estimate $\hat{\theta}(k)$ of the true parameter vector $\theta^{0}$ is updated on the basis of an updating law. It is a member of the set $\hat{\mathcal{G}}(k)$.

Check Strong Robustness: this test indicates when to switch to the second phase of the algorithm. Strongly robust sets of systems are defined as follows.

Definition 3.1 (Strongly robust sets). $\hat{\mathcal{G}}(k)$ is said to be strongly robust if for any systems $P_{1}, P_{2} \in \hat{\mathcal{G}}(k)$, the controller based on the system $P_{1}$ also stabilizes $P_{2}$.

\subsubsection{Control phase}

Design a controller: at each iteration, the controller is designed on the basis of the updated model and the control objective verifying Assumption 2.2, using the certainty equivalence principle. This control phase is fairly standard and is described in [5] in the case of pole placement. 


\section{Analysis of the adaptive system}

The purpose of this section is to study some properties of the systems resulting from the algorithm given in Figure 1, independently of the computational issues, i.e., assuming the various components presented in section 3 to be feasible.

\subsubsection{Neutrality}

The proposed scheme has the property of Neutrality, i.e, in case the present uncertainty set is not falsified by the new measurement, the model is not subject to any update. In particular, if at the time $k$ the model error is strictly zero, then there is no better estimate model than the actual one. Obviously, the case where no update occurs does not imply that the model equals the true system, but it means that the newly observed data do not give any new information with respect to the identification process.

\subsubsection{Convergence of $\hat{\mathcal{G}}(k)$ to a strongly robust set}

The notion of strong robustness is introduced in [2]. In particular, the existence of strongly robust sets in $\mathcal{P}_{n}$ is shown. We now recall the following theorem:

Theorem 4.1 (Existence of strongly robust sets). For any system $P^{0}$ in $\mathcal{P}_{n}$, there exists an open neighborhood of $P^{0}$ contained in $\mathcal{P}_{n}$ which is strongly robust.

In the sequel, the smaller the uncertainty set $\hat{\mathcal{G}}(k)$ is, the more it is likely to be strongly robust. Theorem 4.1 induces the following result:

Theorem 4.2. If the identification input sequence $\{u(k)\}$ is such that $\lim _{k \rightarrow \infty} \hat{\mathcal{G}}(k)=\left\{\theta^{0}\right\}$, then $\hat{\mathcal{G}}(k)$ becomes strongly robust in a finite number of iterations.

Therefore, if $\lim _{k \rightarrow \infty} \hat{\mathcal{G}}(k)=\left\{\theta^{0}\right\}$, then a strongly robust uncertainty set is identified in finite time, i.e., the system does not stay in the identification phase indefinitely long.

Remark 4.1. Note that whether a set of systems is strongly robust or not depends on the control objective, i.e., a given uncertainty set can be strongly robust with respect to some control objectives and not strongly robust with respect to some others. This implies that the rate of convergence of the uncertainty set to a strongly robust set depends on the control objective itself. This issue is investigated in Section 6 .

\subsubsection{Convergence of the model to the real system}

It follows from $(3.6)$ that at any time $k, \hat{\mathcal{G}}(k) \subset \hat{\mathcal{G}}(k-1)$. A natural choice is thus to generate the new estimate parameter at time $k$ as the orthogonal projection of the previous estimate $\hat{\theta}(k-1)$ on the set $\hat{\mathcal{G}}(k)$. This leads to the following update law:

$$
\hat{\theta}(k)=\arg \min _{\theta}\left\{(\theta-\hat{\theta}(k-1))^{T}(\theta-\hat{\theta}(k-1)):\left|y(i)-\phi^{T}(i) \theta\right| \leq d, \forall i \leq k\right\} .
$$


The convergence of $\hat{\theta}(k)$ to the true parameter vector is not guaranteed. However the construction (4.7) provides the following results:

Property 4.1. The model error sequence $\left\{\hat{\theta}(k)-\theta^{0}\right\}$ is bounded and non increasing:

$$
\left\|\hat{\theta}(k)-\theta^{0}\right\| \leq\left\|\hat{\theta}(k-1)-\theta^{0}\right\|, \forall k
$$

and is asymptotically slow, i.e,

$$
\lim _{k \rightarrow \infty}\left(\left\|\hat{\theta}(k)-\theta^{0}\right\|-\left\|\hat{\theta}(k-1)-\theta^{0}\right\|\right)=0 .
$$

From Theorem 4.2, if the identification input $u$ is such that $\lim _{k \rightarrow \infty} \hat{\mathcal{G}}(k)=\left\{\theta^{0}\right\}$, then the asymptotic convergence of the model $\hat{\theta}(k)$ to the real system $\theta^{0}$ is guaranteed i.e., $\lim _{k \rightarrow \infty} \hat{\theta}(k)=\left\{\theta^{0}\right\}$. However we emphasize that for the control purpose, there is no need to stay in the identification phase indefinitely long.

\subsubsection{Stabilizing controller}

In the second phase of the algorithm, control is key. Similar as in the classical adaptive approach, a certainty equivalence type of strategy is adopted. Each new i/o measurement allows to update the uncertainty set and therefore the model. Based on this model, a controller is designed for the real plant to be controlled. Strong robustness of $\hat{\mathcal{G}}(k)$ guarantees that this controller does not destabilize the true plant. Moreover, since at each time the unfalsified set is a subset of the previous one, strong robustness is preserved at any future time. This ensures the true closed-loop control system to be stable at any time.

\subsubsection{Control performance}

Intuitively, the main advantage of the adaptive control based on strong robustness is expected to occur in the first phase. Indeed, the crucial difference between our approach and the classical one is that the case where a destabilizing controller would be applied to the true plant is not allowed. Hence, the transient behavior is expected to be improved. On the contrary, the asymptotic behavior is expected to be the same as in classical adaptive control [5].

The case $n=1$ is addressed in [3] where some simulations support these intuitive results. Moreover, in the case of first order systems and for any pole placement in a stable closed-loop pole, we can prove the following result:

if the input sequence $\{u(k)\}$ is such that $\hat{\mathcal{G}}(k)$ is strongly robust in finite time, then we can find at least one system in $\mathcal{P}_{n}$ for which the classical adaptive pole assignment exposed in [5] yields arbitrarily bad transients in relation to those obtained with our approach.

A similar analysis of the algorithm for higher order systems is in progress and is part of our further work. After having discussed the main properties of the adaptive system based on strong robustness, we now see how its various components can be computed. 


\section{Computational aspects}

\subsection{Computation of the unfalsified sets}

The issue of computing the set $\hat{\mathcal{G}}(k)$ of parameters $\theta$ compatible with a data set on the basis of a known bound measurement error has received a good deal of attention [10], [12], [15], [16]. Practically, the polyhedron $\hat{\mathcal{G}}(k)$ is computed as the intersection of $2 k$ half spaces in the parameter space defined by equations of the form:

$$
\begin{aligned}
& y(i)-\phi^{T}(i) \theta \leq d, \quad \forall i \leq k \\
& y(i)-\phi^{T}(i) \theta \geq-d, \quad \forall i \leq k .
\end{aligned}
$$

$\hat{\mathcal{G}}(k)$ is usually defined either by its faces or by its vertices. Nevertheless, for large data sets its shape might be extremely complex. For this reason, we prefer to compute an outer bounding set of $\hat{\mathcal{G}}(k)$ having a much simpler shape, mainly an orthotopic or ellipsoidal set [6], [13]. By conservativeness, if this outer bounding set is strongly robust then $\hat{\mathcal{G}}(k)$ is also strongly robust.

\subsection{A priori knowledge on the measurement error}

Although many approaches in parameter estimation theory have been derived assuming some statistics on the measurement error ([7], [11]), we only suppose that a conservative bound $d$ on $\delta$ is available. Our first motivation is that the computation of the unfalsified set would not be feasible if we did not suppose a bound to be known. A second motive for this choice is that for small data sets, stochastic assumptions may not be justified. Finally, this approach has also the merit to include the case where the measurement error is correlated to the regression vector.

Remark that $\hat{\mathcal{G}}(k)$ is more likely to be strongly robust if the ratio between the bound $d$ on the measurement error and the norm of the regressor vector is small. This is because the volume of the set $\hat{\mathcal{G}}(k)$ depends on this ratio. Hence we expect the first phase of our algorithm to be completed faster for less conservative bound $d$ on the measurement error.

\subsection{Design of the identification input}

The task of the identification phase is to lead to a strongly robust set $\hat{\mathcal{G}}(k)$ as fast as possible. Hence, the question we raise is: how to compute an input sequence $\{u(k)\}$ such that the volume of $\hat{\mathcal{G}}(k)$ is uniformly decreasing with time? If closed-loop design is adopted, $u$ is proportional to the regressor vector according to a feedback law of the form $u(k)=g(k)^{T} \phi(k)$, with $g(k) \in \mathbb{R}^{2 n}$. Then the problem is to find a gain sequence $\{g(k)\}$ such that the volume of $\hat{\mathcal{G}}(k)$ is uniformly decreasing. This problem is solved in the case $n=1$ in [3] and is under investigation for higher orders. 


\subsection{Test on strong robustness}

In [4], we propose a test for strong robustness. Rather than directly testing the strong robustness of $\hat{\mathcal{G}}(k)$, we first enclose this set in an outer bounding box $\mathcal{B}(k)$ of systems in $\mathcal{P}_{n}$. We then proceed according the three following steps. We first compute the set $\phi(\mathcal{B})(k)$ in the controller space containing the controllers based on all the systems in $\mathcal{B}(k)$. Then we build the set $\chi(\mathcal{B})(k)$ of characteristic polynomials obtained from the association of any system in $\mathcal{B}(k)$ controlled with any controller in $\phi(\mathcal{B})(k)$. Finally we test the stability of any polynomial in $\chi(\mathcal{B})(k)$ using the Kharitonov's theorem. If any polynomial in $\chi(\mathcal{B})(k)$ is Schur stable, then $\mathcal{B}(k)$ is strongly robust. By conservativeness, then $\hat{\mathcal{G}}(k)$ is also strongly robust. Figure 2 illustrates these three steps.

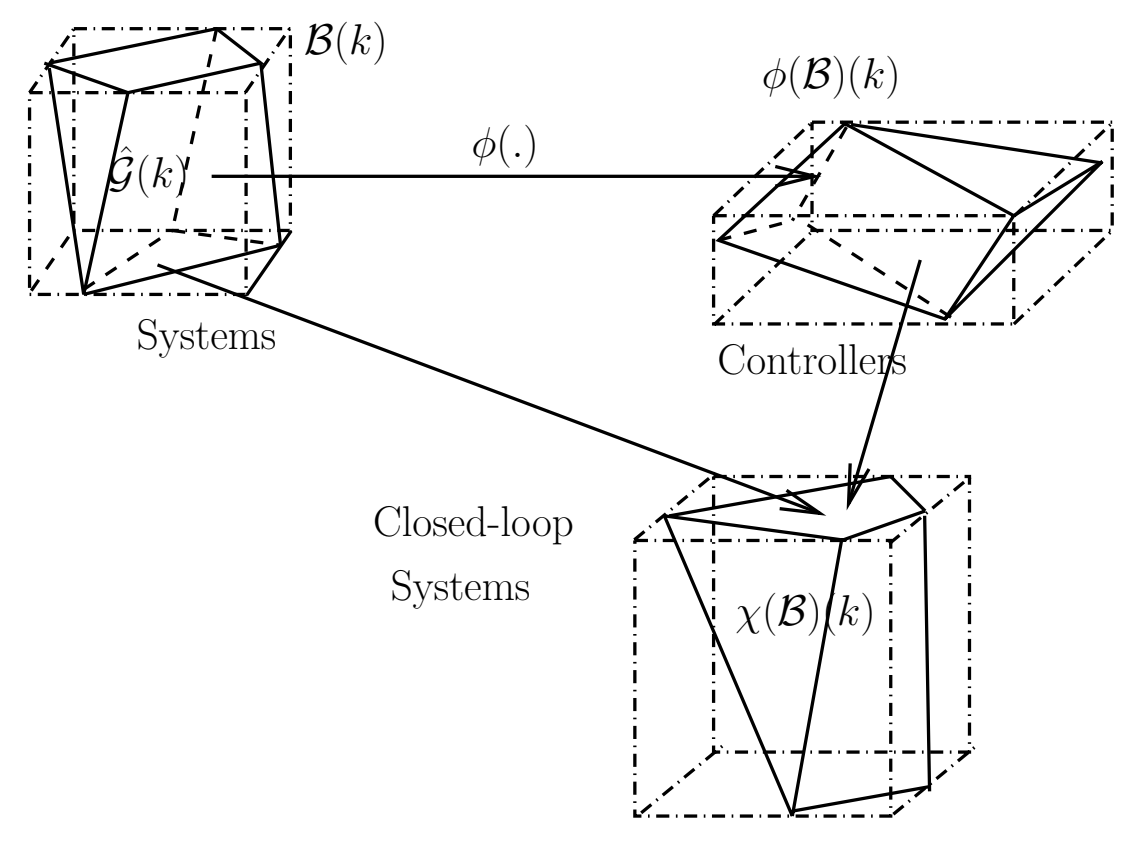

Figure 2: Test on strong robustness.

\subsection{Design a controller}

In the second phase, the basic idea is to switch gradually from the learning phase to the control phase, i.e., we would like to maintain the identification procedure while improving the performance of the controlled system. The question is now: how to choose the input such that at time $k$ the controlled system shows better performance than at time $k-1$ but also such that $\hat{\mathcal{G}}(k) \neq \hat{\mathcal{G}}(k-1)$ ? This problem, which is part of our further work, also raises the following question: since our final objective is to control the initial system, how can we check in which extent the control objective is reached? In general, to answer this question is not easy. Nevertheless, if our control objective is pole placement, we might check whereas 
the control objective is reached simply by comparing the poles of the adaptive system with the desired poles.

We now see in which measure our results depend on the choice of the control objective.

\section{Weak strong robustness}

In Remark 4.1 we note that the outcome of the strong robustness test depends on the control objective. Otherwise stated, a set of systems which is not strongly robust with respect to a control objective might be strongly robust if we update the control objective. Hence, the performance of our algorithm depends on the desired control objective. This might be of interest if the control objective is defined as a collection of control objectives rather than being defined uniquely. Within these control objectives, there exists one for which the algorithm is completed in a minimal time.

Therefore, in order to obtain a strongly robust uncertainty set as fast as possible, we suggest to update the control objective each time a new uncertainty set is identified as the "closest" control objective to the desired control objective leading to a strongly robust set, where "closest" should be specified. We define a weakly strongly robust set of systems as follows:

Definition 6.1. A set $\mathcal{S} \subset \mathcal{P}_{n}$ is said to be weakly strongly robust if there exists a control objective such that $\mathcal{S}$ is strongly robust with respect to this control objective.

Any control objective solution of this problem is then said to be weakly achievable. Remark that the distance between the class of weakly achievable control objectives and the desired control objective is likely to decrease iteratively if the uncertainty set shrinks with time.

Our idea is then to optimize the control objective amongst the class of control objectives that are weakly achievable with respect to the actual uncertainty set so that a strongly robust set is found in a minimal time. The controlled system is expected to show better transients, at the cost of an additional computational load. The analysis of the multi-phase adaptive control based on weak strong robustness is in progress.

\section{Strong robustness: a refined definition}

In this section, we briefly discuss a further refinement of the definition of strongly robust sets. We first explain the motivation for this refinement.

The basic motivation for introducing adaptive control systems based on strong robustness is to ensure that at each time the controller $\phi(\hat{\theta}(k))$ based on the model $\hat{\theta}(k)$ is applied on the true plant, the resulting (time-invariant) closed-loop system $\left(\theta^{0}, \phi(\hat{\theta}(k))\right)$ is asymptotically stable. However, this does not imply that the time-varying system $\left(\theta^{0}, \phi(\hat{\theta}(k))\right)$ itself is asymptotically stable. Note that this is not an issue in the case of first order systems. We propose the following refined definition for strongly robust sets: 
Definition 7.1 (Strongly robust sets). $\mathcal{S} \subset \mathcal{P}_{n}$ is said to be strongly robust if for any system $P \in \mathcal{S}$ and any sequence of systems $\left\{P_{k}\right\}_{k \in \mathbb{N}} \subset \mathcal{S}$ the time-varying system $\left(P, \phi\left(P_{k}\right)\right)$ is asymptotically stable, where $\phi\left(P_{k}\right)$ denotes the controller based on $P_{k}$.

From Definition 7.1 we derive the following sufficient test for strong robustness.

Theorem 7.1. Let set $\mathcal{S}$ be a subset of $\mathcal{P}_{n}$. Given any system $\theta^{0} \in \mathcal{S}$, we denote $\left(A^{0}, b^{0}\right) a$ state space representation of $\theta^{0}$, replacing (2.1) by

$$
\phi(k+1)=A^{0} \phi(k)+b^{0} u(k) .
$$

We moreover denote by $f\left(\theta^{0}\right)$ the controller based on $\theta^{0}$, so that the corresponding control law is described by

$$
u(k)=f\left(\theta^{0}\right) \phi(k) .
$$

$\mathcal{S}$ is strongly robust if the following equation holds:

$$
\forall \theta_{1}, \theta_{2} \in \mathcal{S},\left\|f\left(\theta_{2}\right)-f\left(\theta_{1}\right)\right\| \leq r_{A_{1}+b_{1} f\left(\theta_{1}\right)}^{\mathbb{C}},
$$

where $r_{A_{1}+b_{1} f\left(\theta_{1}\right)}^{\mathbb{C}}$ denotes the complex stability radius of the closed-loop system $\left(\theta_{1}, \phi\left(\theta_{1}\right)\right)$ with respect to the perturbation structure $\left(b_{2}, I_{n}\right)$ as it is defined in [9].

\section{Conclusion}

This paper introduces the structure of a two-step adaptive control system based on a criterion deciding when to put most of the effort on identification or on control. This criterion consists in checking whether the parameter uncertainty set is such that the controller based on any model taken in this set leads to a stable system when applied to any other model in this set. Our motivation is to avoid the case where a destabilizing controller would be applied to the plant to be controlled. The crucial issue is how to compute an identification input sequence ensuring that the uncertainty set is shrinking with time, so that in a minimal time the strong robustness condition is verified? We emphasize that even if there exists a time at which strong robustness is reached, this time cannot be predicted since it depends on the unknown system to be controlled. In that sense, this defines an ill-posed problem, characteristic of any adaptive approach. For this reason, we have to assume that a large, even infinite, observation set of $\mathrm{i} / \mathrm{o}$ data is available, although this is a quite restricting assumption.

This paper intends to describe the general scheme of adaptive control based on strong robustness, whereas a complete analysis of the algorithm is in progress.

\section{References}

[1] R.R. Bitmead, Iterative control design approaches, Proc. 12th IFAC World Congress, Sydney, Australia, Vol. 9, 381-384, 1993. 
[2] M. Cadic, J.W. Polderman, Strong Robustness in Adaptive Control, Proc. 4th NCN Workshop, Sheffield, UK, 2001, to appear.

[3] M. Cadic, I.M.Y. Mareels and J.W. Polderman, Self-tuning and Strong Robustness: the First Order Case, IEEE Conf. Decision and Control, Las Vegas, USA, 2002, submitted.

[4] M. Cadic and J.W. Polderman, Strong Robustness of Boxes of Systems: a Kharitonovlike test, Eighth International Mechatronics Forum, Enschede, The Netherlands, 2002, submitted.

[5] I.M.Y. Mareels and J.W. Polderman, Adaptive Systems: An Introduction, Birkhäuser, Boston, pp. 103-135, 1996.

[6] E. Fogel, Y.F. Huang, On the value of Information in System Identification-Bounded Noise Case, Automatica, Vol. 18, No. 2, 229-238, 1982.

[7] D. Graupe, E. Fogel, A unified sequential identification structure based on convergence considerations, Automatica, Vol. 12, 53, 1976.

[8] R.G.R.J.P. Schrama, P.M.J. Van den Hof, Approximate identification with closed-loop performance criterion and application to LQG feedback design, Automatica, Vol. 30, 679-690, 1994.

[9] D. Hinrichsen, A.J. Pritchard, Robustness measures for linear state space systems under complex and real parameter perturbations, Perspectives in Control Theory, Proc. Sielpa Conference, Sielpa, Poland, Birkhäuser, 1988.

[10] A.V. Kuntsevich, Set-membership identification for robust control, CESA'96 IMACS Multiconference, Lille, France, Vol. 2, 1168-1172, 1996.

[11] I.D. Landau, Unbiased recursive identification using model reference adaptive techniques, IEEE Trans. Aut. Control, AC-21, 194, 1976.

[12] J. Norton, S.H. Mo, Parameter bounding from time-varying systems, Math. Comput. Simulation, Vol. 32, 527-534, 1990.

[13] J.P. Norton, Bounding Techniques for Model-Structure Selection, Proc. International Workshop on Robustness in Identification and Control, Torino, Italy, 59-65, 1988.

[14] S.M.Veres, Iterative Identification and Control Redesign via Unfalsified Sets of Models; a Basic Scheme, International Journal of Control, Vol. 72, 887-903, 1999.

[15] S.M. Veres et al., (1993-98). The Geometric Bounding Toolbox, Version 5.2. MATLAB/SIMULINK Connections Catalog of MathWorks Inc., Licensed by The University of Birmingham, Web: http//www.eee.bham.ac.uk/gbt. 
[16] E. Walter, H. Piet-Lahanier, Estimation of parameter bounds from bounded-error data: a survey, Math. Comput. Simulation, Vol. 32, 449-468, 1990. 